\title{
Prospects and status of the physics run of the NINJA experiment
}

\author{
Takahiro Odagawa* \\ for the NINJA collaboration \\ Kyoto University \\ E-mail: bdaqawa.takahiro.57wdst.kyoto-u.ac.jp
}

\begin{abstract}
Neutrino-nucleus interaction is a major source of the systematic uncertainty for neutrino oscillation experiments. The NINJA experiment aims to measure the neutrino-water interactions precisely with a nuclear emulsion detector called an Emulsion Cloud Chamber (ECC). Nuclear emulsions have sub-micron position resolution and allow us to detect short tracks of low momentum secondary charged particles such as protons.

In the NINJA experiment, a muon detector is placed downstream of ECC because it cannot identify muons from $v_{\mu}$ charged current interactions by itself. In contrast with its good position resolution, the nuclear emulsion doesn't have timing information and an enormous number of tracks are accumulated in the detector during the whole experiment. Therefore, the position resolution of the muon detector is not enough to connect the muon tracks to ECCs one by one. The NINJA experiment solves this problem by using a scintillator tracker between the muon detector and ECC, which has equivalent timing resolution and better position resolution.

The NINJA experiment is planning a physics run with a $75 \mathrm{~kg}$ water target from November 2019. Since the target mass is larger than previous runs, a larger tracker covering $1 \mathrm{~m} \times 1 \mathrm{~m}$ area is needed.

In this paper, we will show the status of preparation for the physics run, especially about development of the new scintillator tracker.
\end{abstract}

The 21st international workshop on neutrinos from accelerators (NuFact2019)

August 26 - August 31, 2019

Daegu, Korea

${ }^{*}$ Speaker. 


\section{NINJA Experiment}

NINJA (Neutrino Interaction research with Nuclear emulsion and J-PARC Accelerator) is an experiment aiming to measure the cross section of neutrino-nucleus interaction exclusively and precisely. The neutrino-nucleus interaction is one of the major sources of the uncertainty in neutrino oscillation experiments such as the $\mathrm{T} 2 \mathrm{~K}$ experiment[四]. Recently, interaction of neutrinos and correlated nucleons, especially $2 \mathrm{p} 2 \mathrm{~h}$ (2-particle 2-hole) interaction, is getting attention for solving the discrepancy between experimental data and CCQE (Charged Current Quasi Elastic) estimation[[], [3]. To obtain purer $2 \mathrm{p} 2 \mathrm{~h}$ samples, higher position resolution is required because protons from the $2 \mathrm{p} 2 \mathrm{~h}$ interaction have low momentum down to $200 \mathrm{MeV} / \mathrm{c}$ and their tracks are very short. However, in conventional scintillator-based detectors, position resolution is insufficient to detect short tracks of such protons.

To achieve higher position resolution, the NINJA experiment uses emulsion films in its detector. The emulsion film consists of gel and dispersed $\mathrm{AgBr}$ (silver bromide) crystals. When a charged particle passes through the emulsion films, it ionizes $\mathrm{AgBr}$ along its path. Then after the development procedure, silver grains can be seen as the track of the particle. Thus, the position resolution of the emulsion film has the potential to reach the diameter of one silver grain.

So far, the NINJA experiment has conducted several test runs in J-PARC (Japan Proton Accelerator Research Complex) [四, 国]. From November 2019, the NINJA experiment will start its physics run. In the physics run, we will have $\sim 5.0 \times 10^{20} \mathrm{P}$. O. T. (Protons on Target) neutrino beam with $75 \mathrm{~kg}$ water as a target material, corresponding to almost $3000 \mathrm{CC}$ (Charged Current) events inclusively. Using these large statistics, the NINJA experiment aims to provide various information of the neutrino-water interaction such as $2 \mathrm{p} 2 \mathrm{~h}$ in the T2K energy region $(\sim 1 \mathrm{GeV})$.

\section{Detectors}

The NINJA experiment has three kinds of detectors in its setup as shown in Figure $\mathbb{W}$.

In the most upstream, Emulsion Cloud Chambers (ECCs) are set as target and vertex tracking detectors. On the most downstream, there is a Muon Range Detector (MRD) called Baby MIND (Magnetized Iron Neutrino Detector) to obtain the kinematics of muons going out of ECCs. Baby MIND has a sandwich structure of plastic scintillator tracking planes and magnetized iron plates.

Because emulsion films don't have timing information, all tracks during the beam exposure are accumulated in them. The position resolution of Baby MIND $(1-20 \mathrm{~cm})$ is insufficient to discriminate muon tracks of interactions in ECCs from ones of background and to connect them to ones detected by Baby MIND. Thus, in the NINJA experiment, detectors for track connection are placed between ECCs and Baby MIND.

There are two kinds of detectors for this purpose. One is an emulsion shifter which consists of moving and fixed emulsion films. It provides rough timing information (several hours - a few days) and very precise position information of the emulsion films. The other is a scintillator tracker which consists of plastic scintillator bars, WLS (wavelength shifting) fibers, and MPPCs (Multi Pixel Photon Counters). This provides beam bunch timing information and better position resolution (a few $\mathrm{mm}$ ) than Baby MIND. 
So far, we have constructed and prepared these detectors for the physics run. From the late October 2019, the NINJA experiment starts the installation of all detectors into the J-PARC NM (Neutrino Monitor) building.
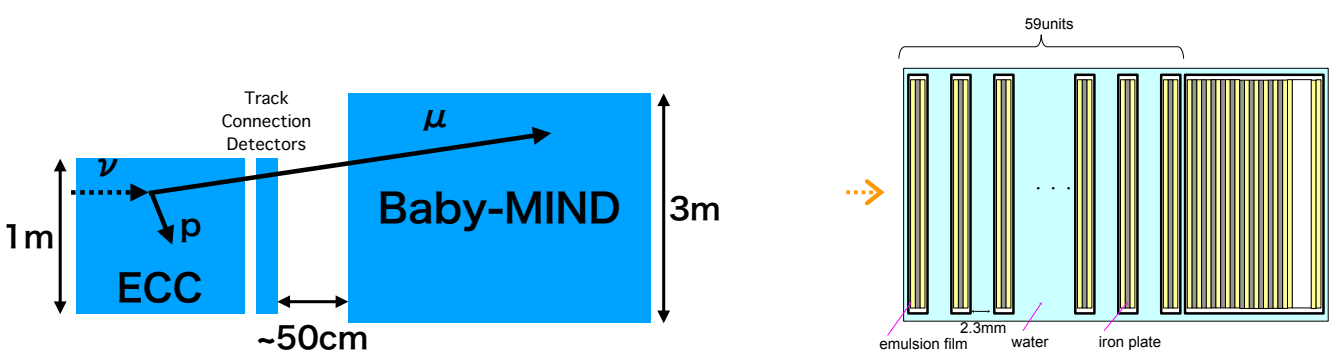

Figure 1: Detector setup for the physics run: ECCs Figure 2: Structure of ECC used in the physics run: are placed in the most upstream and Baby MIND is 58 water layers and 59 tracking layers (consisting in the most downstream. Between them, two types of two emulsion films and an iron plate) are alterof track connection detectors are placed. natively set in a water tank.

\subsection{ECC (Emulsion Cloud Chamber)}

An ECC has a sandwich structure of tracking layers and water layers as shown in Figure $\square$. Charged particles from neutrino interactions in the water layers coming to the emulsion films are tracked by the tracking layers. Each tracking layer has two $350 \mu \mathrm{m}$ emulsion films for tracking on both sides of one $500 \mu \mathrm{m}$ iron plate for momentum measurement.

The preparation of the emulsion films for the physics run has been successfully done in Nagoya University. Those films are now waiting for the detector assembly.

\subsection{Emulsion Shifter}

The emulsion shifter consists of emulsion films attached on two moving walls and one fixed wall. Each moving wall shifts $2 \mathrm{~mm}$ every 4 hours and 3.5 days respectively and connecting tracks in the moving walls to ones in the fixed wall will provide timestamp to each muon track.

The construction and preparation of the emulsion shifter has been also conducted in Nagoya University. Performance test of the shifter with comic muons is ongoing above ground.

\subsection{Scintillator Tracker}

The scintillator tracker has 248 staggering scintillator bars as shown in Figure [3. Using not

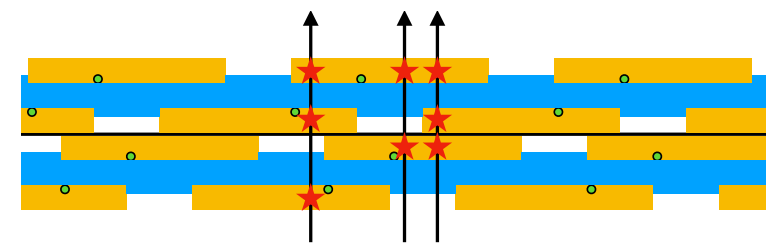

Figure 3: The structure of staggering scintillator bars in the tracker for one $\mathrm{x}$ (or $\mathrm{y}$ ) layer. Each orange rectangle indicates one plastic scintillator bar and blue is a PVC supporting plane. Using hit and unhit information, better position resolution than the width of a scintillator bar can be obtained. 
only hit but also unhit information in each layer can make position resolution around a few $\mathrm{mm}$ which is better than the width of one scintillator bar $(2.4 \mathrm{~cm})$. For example, the left track in Figure 3 makes hits in three layers but not in the second one from the bottom. On the other hand, the middle track makes hits in two layers and no hits in the other layers. Seeing such hit/unhit patterns will distinguish one bar into six segments and thus six times better position resolution can be obtained.

The construction has been done in J-PARC from February to May 2019. First, the fibers are glued on the scintillator bars with optical cement and those bars are put on PVC supporting plates. All fiber edges of readout sides are polished with a diamond cutter and are attached to MPPCs through special optical connectors. After that, one micro coaxial cable is connected to each MPPC through special PCB connectors. Every component was successfully assembled as shown in Figure 团.

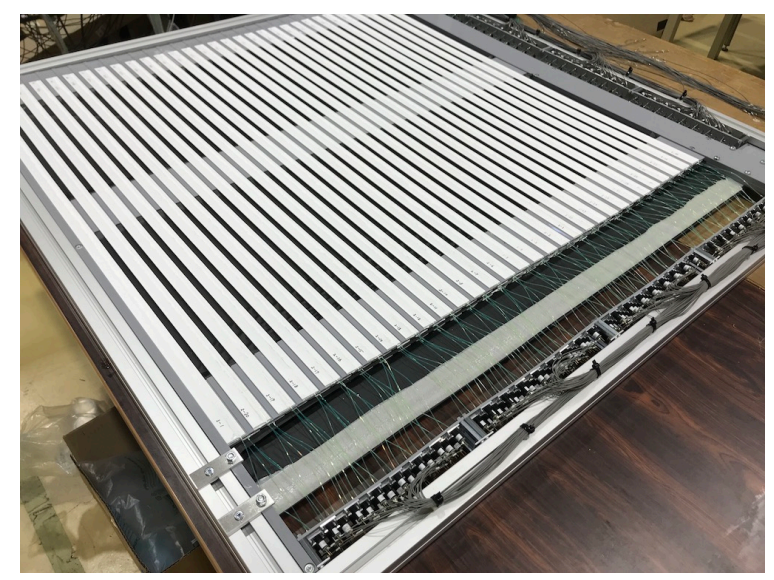

Figure 4: Scintillator tracker after the construction

After the construction, a connection check and a gain calibration with dark current of MPPCs, and light yield measurement with cosmic muons have also been done in J-PARC. As for the connection check, four channels were found to be disconnected. All four channels were recovered by replacing one MPPC, one coaxial cable, and two PCB connectors. After confirming all channels' connection, the light yield for cosmic muons (as MIP) was checked. All channels except six ones show sufficient light yield for cosmic muons. Using the most probable value of the spectra, detection efficiency of each scintillator bar is estimated for 1.5 p.e. threshold. Almost all channels show more than $99 \%$ efficiency for the threshold as shown in Figure 5 .

\section{Prospects for the Physics Result}

Figure 6 shows examples of the expected number of events and spectrum in the next physics run of the NINJA experiment with $5.0 \times 10^{20}$ P. O. T. beam exposure.

Goals of the NINJA experiment are i) to get distributions of multiplicity of charged particles $(p, \pi)$, and their momentum and angle, ii) to measure exclusive neutrino-water cross section, and iii) to constrain models of the $2 \mathrm{p} 2 \mathrm{~h}$ interaction by selecting events with no pion and two protons in their final states $(\mathrm{CC} 0 \pi 2 p)$, which will contain enriched $2 \mathrm{p} 2 \mathrm{~h}$ interaction events. All information will be highly useful to reduce the systematic uncertainty of the T2K experiment. 

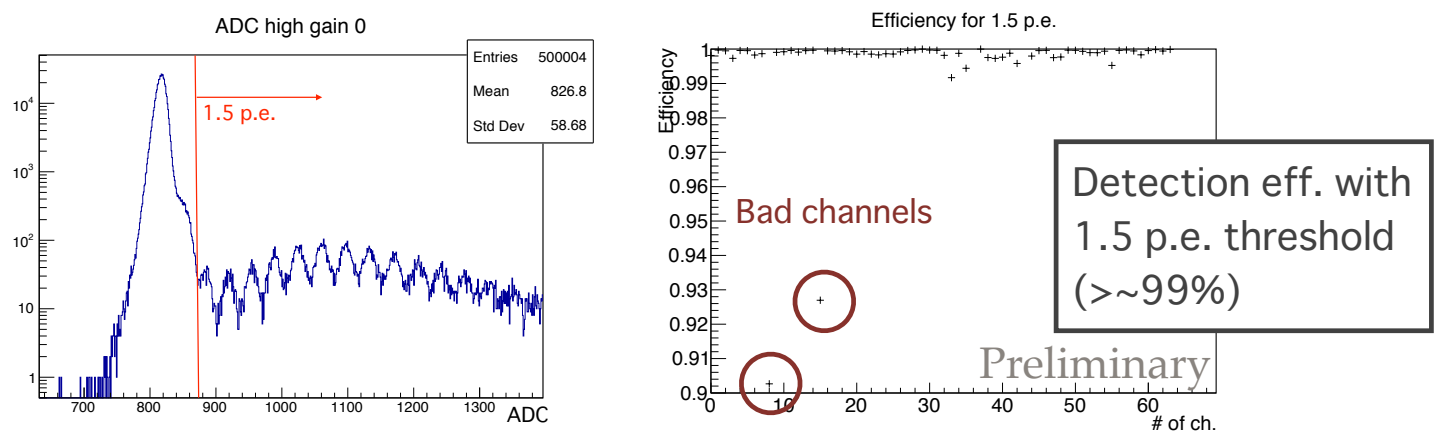

Figure 5: The result of efficiency calculation of the tracker. The left figure shows the spectrum of cosmic muons in one channel. The red line indicates 1.5 p.e. threshold. The right figure is the plot of detection efficiency of 64 channels. Almost all channels show $99 \%$ efficiency except circled channels which show lower light yield.
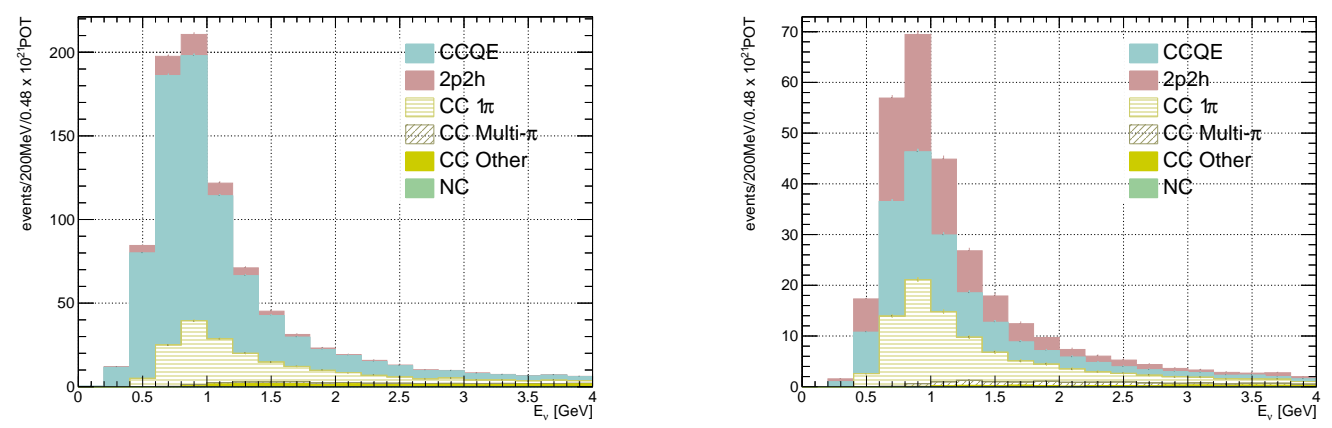

Figure 6: Expected number of events in the next physics run of the NINJA experiment. The left histogram shows $\mathrm{CC} 0 \pi 1 p$ events and the right shows $\mathrm{CC} 0 \pi 2 p$ ones. In the $\mathrm{CC} 0 \pi 2 p$ events, the number of $2 \mathrm{p} 2 \mathrm{~h}$ events is expected to be comparable to the CCQE events.

\section{Conclusion}

In conclusion, the NINJA experiment aims at a precise measurement of the exclusive cross section of neutrino-nucleus interactions with high position resolution of the emulsion films. From November 2019, the NINJA experiment will start its physics run with $75 \mathrm{~kg}$ water target and preparation of the detectors is ongoing in J-PARC. All detectors were successfully constructed, and especially the new scintillator tracker was developed. This new tracker has the structure of mutually staggering scintillators and their hit/unhit information leads to the better position resolution than scintillator width. Using the large statistics, various new information of neutrino-water CC interaction in $1 \mathrm{GeV}$ energy region is expected to be provided by the NINJA physics run.

\section{References}

[1] K. Abe et al. (T2K Collaboration), Phys. Rev. Lett. 121, 171802 (2018).

[2] J. Nieves, I. R. Simo, and M. J. V. Vacas, Phys. Rev. C 83, 045501 (2011).

[3] M. Martini et al., Phys. Rev. C 81, 045502 (2010). 
[4] T. Fukuda et al., Prog. Theor. Exp. Phys. Volume 2017, Issue 6, 063 C02 (2017).

[5] K. Yamada et al., Prog. Theor. Exp. Phys. Volume 2017, Issue 6, 063 H02 (2017). 